\title{
A Bayesian approach to luminescent down-conversion
}

\author{
M. Löning, ${ }^{1, \text { a) }}$ L. Lombez, ${ }^{2}$ J.-F. Guillemoles, ${ }^{1, \text { b) }}$ and D. Suchet ${ }^{1, c)}$ \\ 1) CNRS, Ecole polytechnique UMR 9006, Institut Photovoltaique d'Ile de France (IPVF), \\ Palaiseau, France \\ ${ }^{2)}$ CNRS, LPCNO, Toulouse, France
}

(Dated: December 9, 2020)

Photon conversion embodies a range of promising possibilities in pushing the theoretical Shockley-Queisser efficiency model of classical solar cells. Luminescent downconversion, despite its potential, is held back in practical applications by the difficulty of proper characterization, in no small part because of concurrent luminescent downshifting events. Recent advances have demonstrated the opportunity provided by photon correlation measurement for DC characterization. In this methodological work, we present a general method, based on Bayesian probabilities, for deriving auto-correlation functions analytically. This method is then applied to the five down-conversion mechanisms reported in the literature and successfully tested against numerical simulations. We show that the zero delay auto-correlation function can be the most direct way to demonstrate down-conversion and assess its efficiency. Our analysis offers additionally useful tools for the design of characterization experiments, and emphasizes some universal behavior valid for all reported conversion mechanisms.

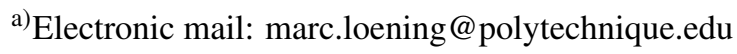

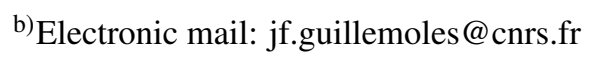

c)Electronic mail: daniel.suchet@polytechnique.edu
} 
A Bayesian approach to luminescent down-conversion

\section{INTRODUCTION}

Applications of photon conversion to photovoltaics (PV) are diverse, promising ${ }^{1-4}$ and well debated $^{5,6}$. Luminescent downshifting (LDS) for example, by which a high-energy photon is transformed into a single lower-energy photon, allows for minimal front-layer losses and improved $\mathrm{EQE}^{2,7-9}$. Up-conversion - where two photons with sub-bandgap energy are combined into a single photon with supra-bandgap energy, ready to be harvested - and down-conversion (DC) or quantum-cutting - where a single high-energy photon, whose excess energy is typically wasted as photo-generated carriers thermalize towards the bandgap, generates two lower-energy photons allowing the creation of multiple electron-hole pairs - both tackle the limit set by the ShockleyQueisser ${ }^{10}$ model for solar cell efficiency ${ }^{11,12}$.

A broad range of materials have been considered as quantum cutters for PV applications, including quantum dots ${ }^{13-16}$, organic dyes ${ }^{17-20}$, rare-earth compounds ${ }^{21-24}$ and more recently perovskite compounds ${ }^{25}$. These different systems perform down-conversion through different microscopic processes, such as singlet fission, resonant energy transfer, impact ionization or cascade emission (see Fig.1). Despite these differences, a critical question for developing innovative converters of any kind is the characterization of the system's DC efficiency, which we define as the probability for a single absorbed photon to trigger the emission of two low-energy photons.

The usual metric to account for conversion efficiency is the photoluminescent quantum yield (PLQY), which can be defined as the ratio between the number of emitted photons and the number of incident (external PLQY) or absorbed (internal PLQY) photons ${ }^{2}$. Both definitions converge towards the same value for ideal absorbers (full absorption in the active material). A PLQY of 200\%, indicating that every high-energy photon generates two low energy photons, indicates an ideal DC efficiency. However, this indicator becomes much more ambiguous in non-ideal systems ${ }^{26,27}$. It is indeed difficult to distinguish between actual DC events, where two photons are emitted from the same initial excitation, from the LDS signal, where low-energy photons are emitted from independent single-photon processes. For instance, a PLQY of $80 \%$ can be attributed to a system with DC efficiency of $40 \%$ without significant LDS, but also to a system with a LDS efficiency of $80 \%$ without any DC, or to any combination of these two extreme situations. To reach an unambiguous conclusion regarding DC, it is therefore necessary to complement these measurements with further analyses such as kinetic models in quantum dots and nanocrystals ${ }^{28,29}$, with additional simulations such as Monte Carlo studies ${ }^{27,30}$ or with supplementary experiments such as magnetic-field depen- 
A Bayesian approach to luminescent down-conversion

dent spectroscopy in organic compounds ${ }^{31,32}$. However, such investigations are highly dependent on the specificity of each DC system, require a solid knowledge of the system's properties and offer only an indirect signature of the DC process.

An interesting opportunity to gain a straightforward and universal access to DC efficiency comes in the form of photon correlations. Indeed, in a DC process, unlike the simpler LDS, photon emissions are expected to occur as pairs of temporally correlated photons. The experimental signature of the resulting bunched behaviour can thus be distinguished from the correlation measurements yielded by the random, independent photon emissions of LDS. While this approach is quite familiar in the field of parametric photonic conversion, it is yet marginally used in the field of photovoltaics, despite very promising results ${ }^{33}$.

In this work, we discuss a powerful framework based on Bayesian statistics to account for down-conversion correlation signal. In a first section, we introduce the usual mechanisms reported in the literature for DC systems, and the Bayesian estimation of the correlation function. We then use this approach to reach an analytical expression for the zero-delay correlation function, which we validated against numerical simulations. This expression offers significant applications for the investigation of down conversion. Most notably, we demonstrate that the correlation function provides a universal and unambiguous signature of down-conversion, even at PLQY below 100\%, and regardless of the microscopic details of the conversion process. Finally, we show that our analysis provides useful tools, such as critical scaling laws, for the design of characterization experiments and allows a quantitative estimation of down-conversion efficiency in favorable cases.

\section{METHODS AND MODELS}

\section{A. Down-converter models}

For present theoretical purposes, down-converters are modeled as a macroscopic number $N$ of identical, independent emission centers. Five typical mechanisms are considered in the literature to account for the microscopic processes resulting in the actual down-conversion of a single absorbed high-energy photon into two emitted low-energy photons ${ }^{3,4}$. These mechanisms can be decomposed in elementary transitions characterized by branching factors and typical lifetimes. Figure 1 shows these schemes and highlights two categories of converters.

In parallel converters (mechanisms $\mathrm{a}, \mathrm{b}$ and $\mathrm{c}$ in Fig. 1), upon the seminal absorption, the 

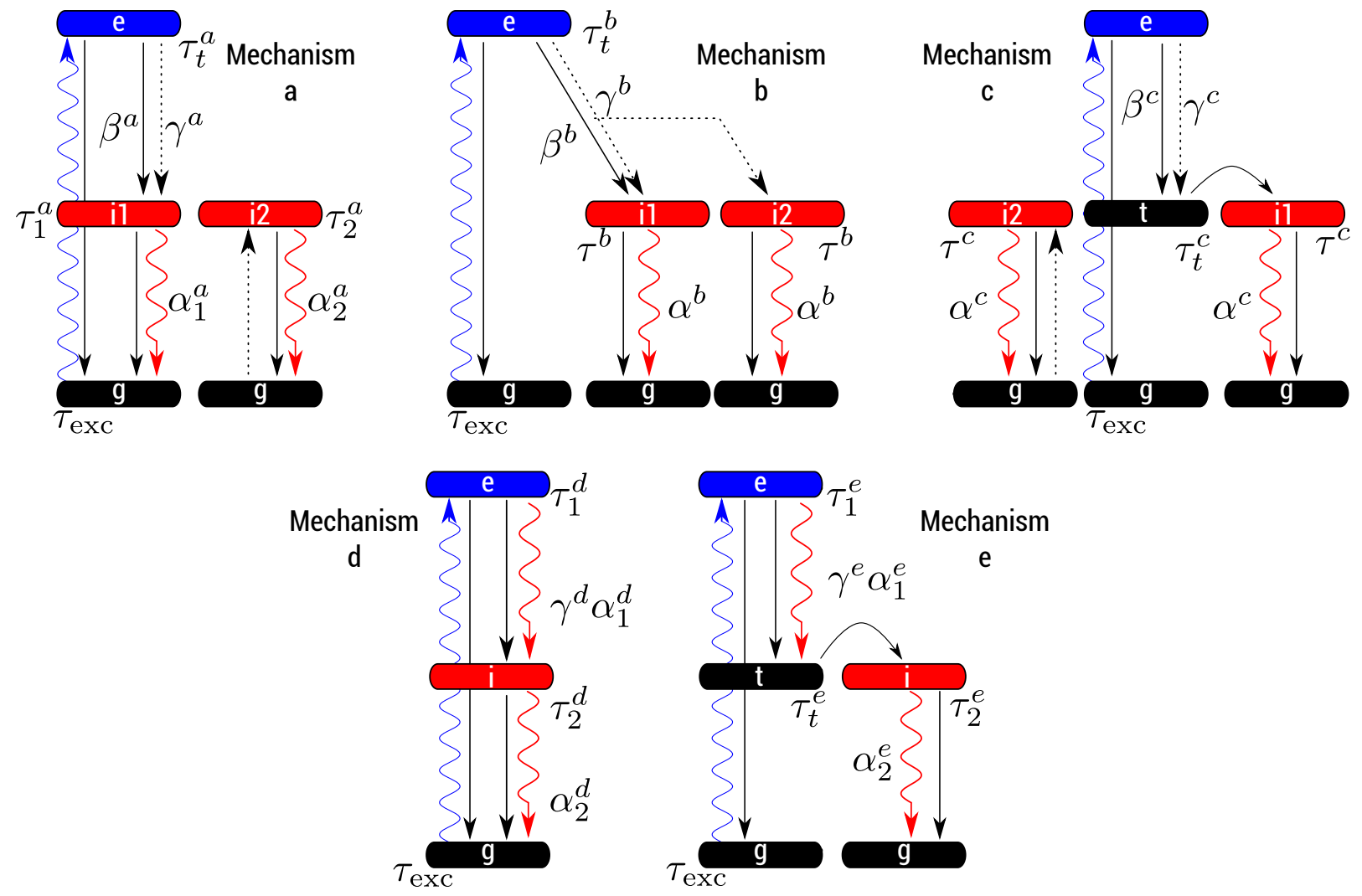

Figure 1. Five mechanisms can be considered for down conversion ${ }^{3,4}$. In this figure, we define basic parameters for each of these mechanisms. Radiative transitions are pictured with colored wavy lines. Energy transfer are represented by two dotted lines corresponding to simultaneous transitions. Non-radiative recombinations are shown with solid black lines. Greek letters $\alpha, \beta$ and $\gamma$ account for the branching ratio of the corresponding transition. Durations $\tau$ indicate the lifetime of the corresponding state.

system can redistribute the imparted energy such that two radiative states are simultaneously populated. This redistribution can occur through a one-step energy transfer (ET) to a neighbouring emitter (mechanism a, reported for $\mathrm{Tm}^{3+}-\mathrm{Yb}^{3+}, \mathrm{Er}^{3+}-\mathrm{Yb}^{3+}$ and $\mathrm{Ho}^{3+}-\mathrm{Yb}^{3+}$ for instance ${ }^{3,4}$ ), or to two acceptors (mechanism $\mathrm{b}$, reported in $\mathrm{Tb}^{3+}-\mathrm{Yb}^{3+}, \mathrm{Tm}^{3+}-\mathrm{Yb}^{3+}$ and $\mathrm{Pr}^{3+}-\mathrm{Yb}^{3+}$ systems ${ }^{3,4}$ as well as in single-fission in organic converters). Another redistribution mechanism can be a twosteps energy transfer (mechanism c), which is largely reported in the literature for $\mathrm{Pr}^{3+}-\mathrm{Yb}^{3+}$, $\mathrm{Er}^{3+}-\mathrm{Yb}^{3+}, \mathrm{Nd}^{3+}-\mathrm{Yb}^{3+}$, or $\mathrm{Dy}^{3+}-\mathrm{Yb}^{3+}$ systems for instance $\left.{ }^{3,4}\right)$.

In series converters (mechanisms d and e in Fig. 1), the system undergoes consecutive relaxations driving him from one radiative state to the next one. This cascade can be obtained 
directly (mechanism d, considered for single-ion emission notably for $\mathrm{Ho}^{3+}, \mathrm{Tm}^{3+}$, or $\mathrm{Er}^{3+}$ based converters $^{3,4}$ ). For the sake of completeness, a cascade involving an intermediate energy transfer can also be considered (mechanism e), but no system has been reported in this category so far.

For simplicity, we will assume that transfer steps from intermediate levels in mechanisms c and e are very fast as compared to the radiative lifetime of the intermediate state, in agreement with the literature ${ }^{30}$. We discuss this assumption below and in the supplementary information.

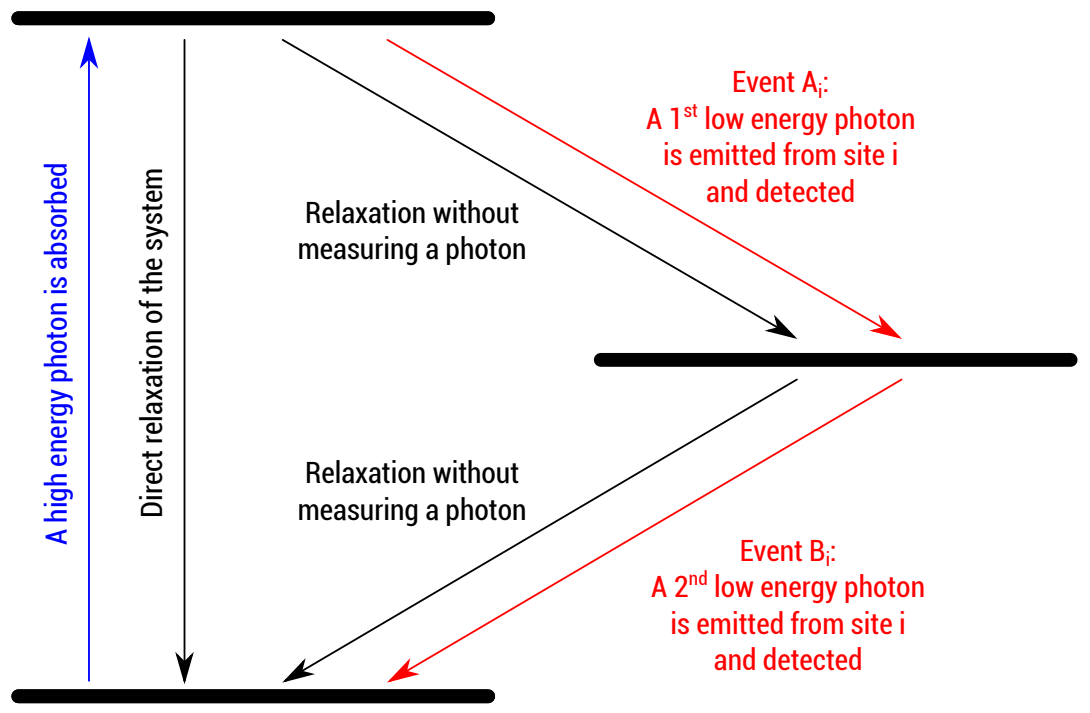

Figure 2. Generic sequence of detection events in mechanisms a-e of figure (1), as measured from a single emission site. The probability for measuring a first photon from any site is $P(\mathrm{~A})=\sum_{i} P\left(\mathrm{~A}_{i}\right)$, where the sum runs over all emitters, and respectively for event $B$.

The aim of our model is to evaluate the auto-correlation function of the detected photons. From the detector perspective, the five aforementioned mechanisms can be reduced to two measurable events (see Fig.3):

- A: a first low-energy photon is emitted from any site and detected.

- B: a second low-energy photon is emitted from any site and detected.

Note however that the probabilities associated to these events are not readily related to those pictured in Fig.1. Indeed, as this simplified scheme neglect internal details of the converter, probabilities for event $A$ and $B$ can depend not only on the current state of the system, but also on its history. Moreover, a sample is usually made of many single emitters, but the correlation between single detection events can be seen when the 2 photons are emitted from the same site. We 
will show is the following sections how these difficulties can be addressed and how the relevant quantities can be expressed as a function of microscopic details.

In some cases, it is possible to distinguish between the two photons resulting from a successful quantum-cutting. For instance possible to resolve them spectrally if the energy is not equally distributed between the two transitions. However, in order to provide the most generic framework, we will consider in the following that the two photons are not distinguishable. In other words, when a photon is detected, we don't know whether it corresponds to an event A or B.

Finally, we extend the scope of the theoretical study by accounting for predictable nonidealities, introduced by the measurement system, which are expected to degrade the correlation signal. To account for the limited optical collection efficiency and the finite efficiency of the detector, we introduce an effective quantum efficiency $Q E$ as the probability for a photon emitted from an emission center to be actually detected. Furthermore, to account for the detection noise, we introduce a dark count rate $\tau_{D}^{-1}$ and consider the corresponding detection event:

$\mathrm{C}:$ a dark count triggers the detector

Further non-idealities are considered and discuss in section IV C.

\section{B. Auto-correlation function}

Down-conversion photons will tend to be detected in pairs, corresponding to successive - and temporally close - emission events $A$ and B. The mathematical tool to describe such photon bunching quantitatively is the optical auto-correlation function ${ }^{34}$, defined as

$$
g^{(2)}(\tau)=\frac{\langle I(t) I(t+\tau)\rangle}{\langle I(t)\rangle\langle I(t+\tau)\rangle},
$$

where $I(t)$ is the light intensity at instant t. In situations considered for down-conversion, $g^{(2)}(\tau)<$ $g^{(2)}(0)$ and we will focus on $g^{(2)}(0)$ which acts as the most practical tool to discuss photon emission statistics. Of course, dependence of $g^{(2)}$ on delay $\tau$ could lead to more information on the processes.

For experimental and numerical purposes, estimations of $g^{(2)}(0)$ are provided by counting the total number $N_{\text {total }}$ of photons detected over the duration $T$ of the experiment and the number $N_{\text {pairs }}$ of events where two photons are detected within the integration time $\Delta T$ of the detector ${ }^{35}$ :

$$
g^{(2)}(0) \underset{\Delta T \rightarrow 0}{=} \frac{T}{\Delta T} \frac{N_{\text {pairs }}}{\left(N_{\text {total }}\right)^{2}} .
$$


A Bayesian approach to luminescent down-conversion

The main idea of this work is to use a Bayesian approach to evaluate this quantity. Bayesian statistics provides indeed a relevant formalism to account for the probability of an event to occur knowing that another event has occurred before. In this framework, the auto correlation function can be expressed as

$$
g^{(2)}(0)=\frac{P(\mathrm{D} \mid \mathrm{D})}{P(\mathrm{D})},
$$

where $P(D)$ is the probability density of a photo-detection from any source (event $A, B$ or $C$ ) and $P(\mathrm{D} \mid \mathrm{D}) \Delta T$ is the conditional probability for a photo-detection to occur within the integration time $\Delta T$ after a first photon has been measured.

\section{Numerical simulation}

To verify our findings, we compare the analytical expression to the results of numerical simulations based on Markov chains. We draw at random the instants for a series of absorptions (typically $10^{6}$ events), according to an exponential law with typical time $\tau_{e} x c$. For each of these absorptions, we draw at random the delay before relaxation takes place, and decay channel. We repeat this process through the cascade, until the system reaches the ground state. We obtain the list of instants at which each radiative transition took place, from which we estimate the intensity measured by the detector as a function of time, taking also dark counts into account. Finally, we evaluate the correlation function of the recorded signal. Figure 3 illustrates the output of such a simulation.

\section{RESULTS}

\section{A. Analytical expression of the zero-delay correlation function $g^{(2)}(0)$}

Considering all events considered in section II A in the Bayesian expression of the correlation function eq.(3) leads to the following expression in the low-excitation regime $\tau_{\text {exc }}>\tau_{2}$ :

$$
g^{(2)}(0)=1+\frac{\sum_{i=1}^{N} P\left(\mathrm{~B}_{i} \mid \mathrm{A}_{i}\right) P\left(\mathrm{~A}_{i}\right)}{P(D)^{2}}
$$

where the sum runs over all possible cascades, $P\left(\mathrm{~A}_{i}\right)$ (resp $P\left(\mathrm{~B}_{i}\right)$ ) is the probability density for detecting a photon resulting from the first step (resp. second step) of the conversion process, $P(\mathrm{D})$ is the probability density for any detection event to occur, and $P\left(B_{i} \mid A_{i}\right) \Delta t$ is the probability for a 


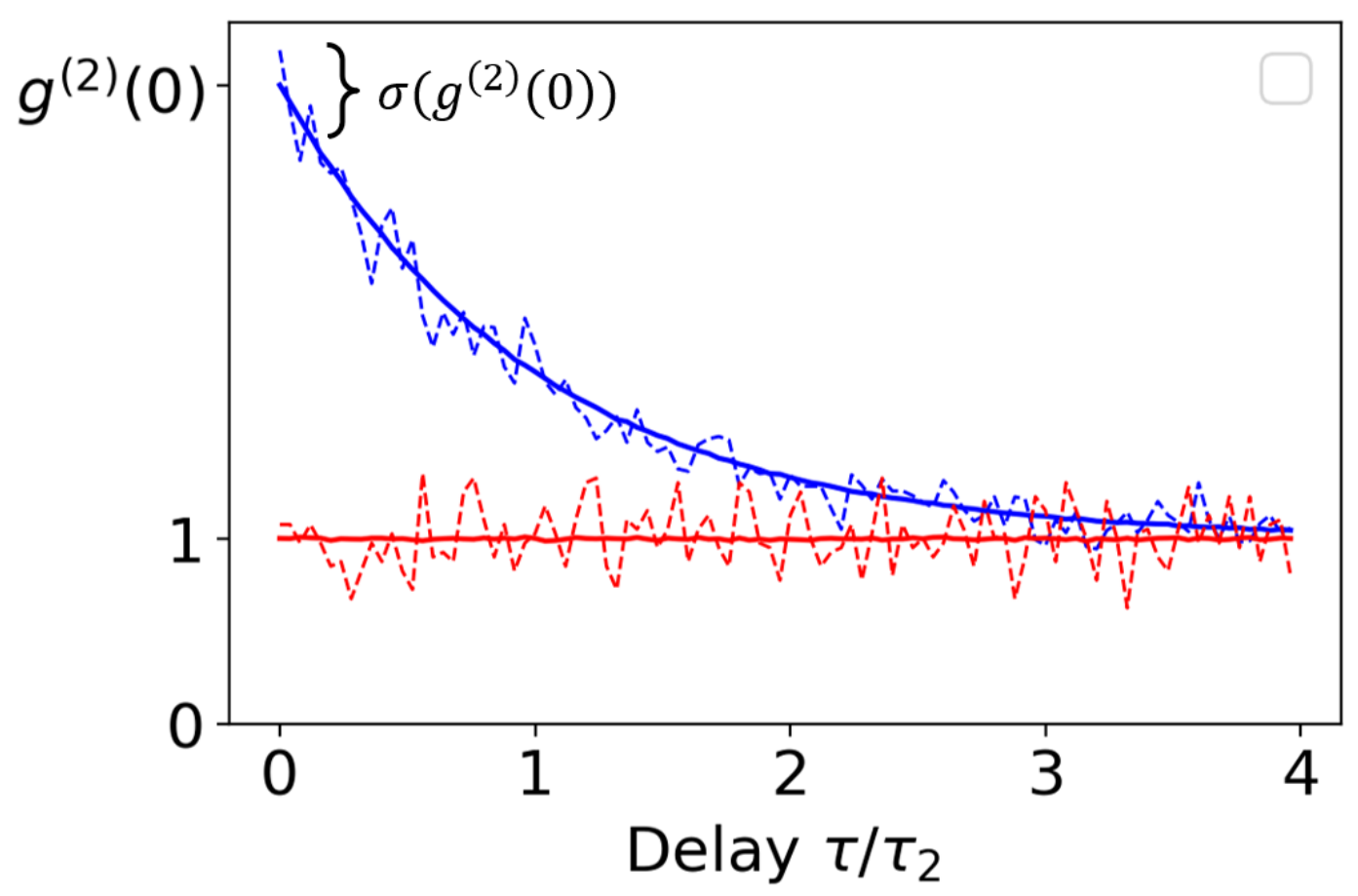

Figure 3. Numerical estimations of the auto-correlation function $g^{(2)}(\tau)$ for the sequential converter (mechanism $\mathrm{d}$ in Fig.1). Blue is for a perfect DC mechanism $(\alpha, \beta, \gamma=1)$, while red is for complete $\operatorname{LDS}(\alpha=0$ and $\beta, \gamma=1$ ). Solid and dotted lines correspond respectively to long and short experiment duration $T$, which will mainly have an impact on the standard deviation $\sigma$ of simulated results. The time evolution of $g^{(2)}(\tau)-1$ and its exponential behavior for mechanisms b-e is derived in appendix.

photon originating from the second step of the cascade to occur within a delay $\Delta t$ after a photon originating from the first step on the same cascade was detected. The derivation of this expression is performed in Appendix A.

This equation has a simple interpretation: $g^{(2)}(0)-1$ is the deviation from a pure random (uncorrelated) process. In the low excitation regime, $\sum P\left(\mathrm{~B}_{i} \mid \mathrm{A}_{i}\right) P\left(\mathrm{~A}_{i}\right) \simeq N \times(P(B \mid A)-P(B)) P(A)$ and this correlation can be expressed as probability of the observed event of a cascade minus the probability of uncorrelated events, normalized by the probability of a double detection event $\left(P(D)^{2}\right)$.

Relating this expression to the microscopic processes described before, we reach the main result of this work:

$$
g^{(2)}(0)=1+\frac{\tau_{\mathrm{exc}}^{-}}{\bar{\tau}} \frac{\eta_{\mathrm{DC}}}{\left(\eta_{\mathrm{LDS}}+\frac{\tau_{e x c}^{-}}{\mathrm{QE} \tau_{D}}\right)^{2}}
$$

where the DC efficiency $\eta_{\mathrm{DC}}$ is the probability for an absorbed photon to give rise to two low- 
A Bayesian approach to luminescent down-conversion

\begin{tabular}{|c||c|c|c|}
\hline Mechanism & $\eta_{\text {DC }}$ & $\eta_{\text {LDS }}$ & $\bar{\tau}$ \\
\hline \hline $\mathrm{a}$ & $\alpha_{1} \alpha_{2} \gamma$ & $\gamma\left(\alpha_{1}+\alpha_{2}\right)+\beta \alpha_{2}$ & $\frac{\tau_{1}+\tau_{2}}{2}$ \\
\hline $\mathrm{b}$ & $\alpha^{2} \gamma$ & $\alpha(2 \gamma+\beta)$ & $\tau$ \\
\hline $\mathrm{c}$ & $\alpha^{2} \gamma$ & $\alpha(2 \gamma+\beta)$ & $\frac{\tau_{t}+\tau}{2}$ \\
\hline $\mathrm{d}$ & $\alpha_{1} \alpha_{2} \gamma$ & $\gamma\left(\alpha_{1}+\alpha_{2}\right)$ & $\tau_{2}$ \\
\hline $\mathrm{e}$ & $\alpha_{1} \alpha_{2} \gamma$ & $\gamma\left(\alpha_{1}+\alpha_{2}\right)$ & $\tau_{2}$ \\
\hline
\end{tabular}

Table I. Table of parameters for equation (5) for the cases presented in figure (1 a-e). It is assumed here that transfer times are very short in case $\mathrm{c}$ and $\mathrm{e}$.

energy photons, the PL quantum yield $\eta_{\mathrm{LDS}}$ is the average number of low-energy photons emitted for each absorbed photon, and $\bar{\tau}$ is the typical time separating the two emission processes. Those material properties characterize the system studied in our model. The other parameters in equation (5) characterize the experimental setup: QE is the fraction of emitted photons that are successfully collected and detected, $\tau_{\mathrm{D}}$ is the average time between dark counts. The last quantity, $\tau_{\mathrm{exc}}^{-}=$ $\tau_{\text {exc }} / N$, the average time between two absorption events among all optical centers and is related to both the material system and the experimental conditions as discussed in subsection IV B. The application of this expression to microscopic rates of the five aforementioned mechanisms is shown in Table I.

The application of eq.(5) to a series converter without energy transfer (case d) recovers an expression already reported in the literature, and obtained with a different approach ${ }^{33}$. To our knowledge, this work is the first to establish an analytical expression for the correlation function of the four complementary situations.

We successfully tested these equations against simulation results (Figs.4 and 5), obtaining a very good agreement between theory and simulation without any fitting parameters.

\section{B. Analytical expression of the uncertainty $\sigma\left(g^{(2)}(0)\right)$}

An important limitation of photon correlation measurements is the statistical nature of the method used for estimating $g^{(2)}(0)$. Experimental results will be marred by statistical noise as shown in Fig.3. This uncertainty can be evaluated if we take $g^{(2)}(0)$ as given experimentally by eq. 2 as a random variable. Then, using the following assumptions : (i) that $N_{\text {pairs }}$ is the main 


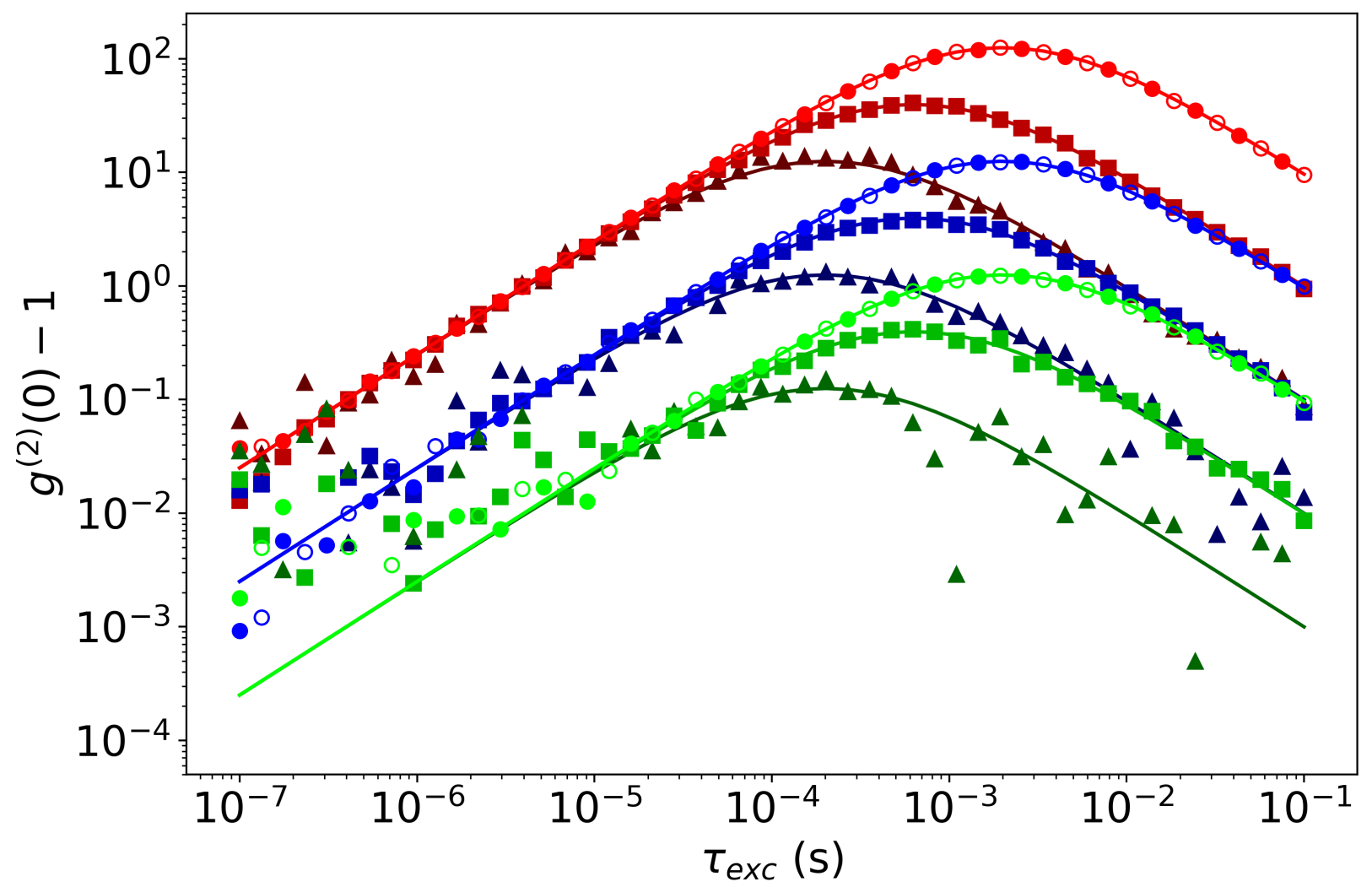

Figure 4. Compared theoretical and simulated results for $g^{(2)}(0)-1$ in the sequential DC case (Fig. 1-d) as a function of $\tau_{e x c}$ for $\tau_{D}=10^{-3}$ s and $\alpha=\beta=\gamma=1$. Each color $(\mathrm{R}, \mathrm{B}, \mathrm{G})$ is associated with a value of $\tau_{2}$ in $\left(10^{-6}, 10^{-5}, 10^{-4}\right)$ s. Shapes $(\boldsymbol{\Lambda}, \boldsymbol{\square}, \bullet)$ indicate the value of $Q E$ in $(1,0.32,0.1)$. Empty $\circ$ and full $\bullet$ represent $\tau_{1}=10^{-5}$ and $10^{-8}$ s respectively.

source of uncertainty (the variance of $N_{\text {total }}$ is neglected), (ii) that $\Delta T<<\tau_{2}$ (needed for accurate determination of $g^{(2)}(0)$ ) and (iii) that $N_{\text {pairs }}$ is Poissonian (no correlation between pair events from different sites), a standard deviation for $g^{(2)}(0), \sigma\left(g^{(2)}(0)\right)$ can be derived analytically to give:

$$
\sigma\left(g^{(2)}(0)\right)=\frac{\tau_{e q}}{\sqrt{T \Delta T}} \sqrt{g^{(2)}(0)}
$$

where $\tau_{e q}=N_{\text {total }} T^{-1}$ is the average time between two detection events $\left(\tau_{e q}^{-1}=\tau_{D}^{-1}+\gamma(\alpha+\right.$ $\beta) Q E \tau_{\text {exc }}^{-1}$ for a series converter for instance). We have also successfully verified this expression against numerical simulations (see Fig. 6 ). 


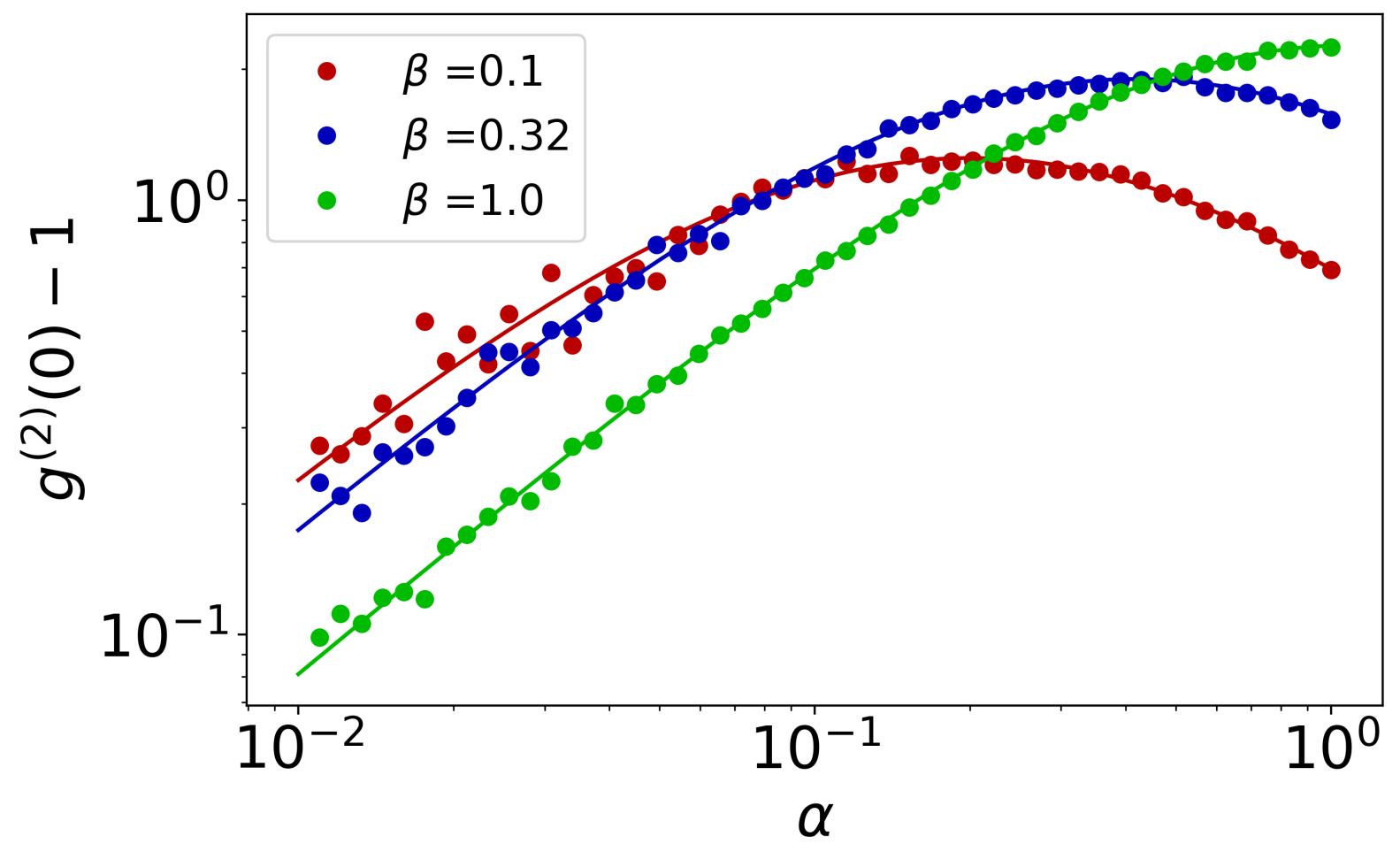

Figure 5. Compared theoretical and simulated results for $g^{(2)}(0)-1$ in the sequential DC case (Fig 1.d) as a function of $\alpha$, for $\beta$ varying in $(1,0.32,0.1)$ and $\tau_{2}=10^{-5}, \tau_{e x c}=10^{-4}, \tau_{D}=10^{-3}$.

\section{DISCUSSION}

\section{A. Universality}

The expression eq.(5) obtained through the Bayesian approach highlights two major properties of the correlation function.

First, the correlation function, unlike PLQY, provides an unambiguous discrimination between LDS and DC. It appears indeed that the quantity $g^{2}(0)-1$ is directly proportional to the DC conversion efficiency, and a non-zero measurement of this quantity offers therefore a clear signature of DC even in systems with limited efficiencies (see Fig. 5).

Second, this expression reveals the universality of the correlation function, as all five schemes pictured in Fig. 1 can be mapped onto a parallel converter (mechanism a). Mechanism b corresponds to a particular case of a with identical properties for both radiative transitions. More surprisingly, a series converter (mechanism d) is formally equivalent to a symmetric parallel con- 
A Bayesian approach to luminescent down-conversion

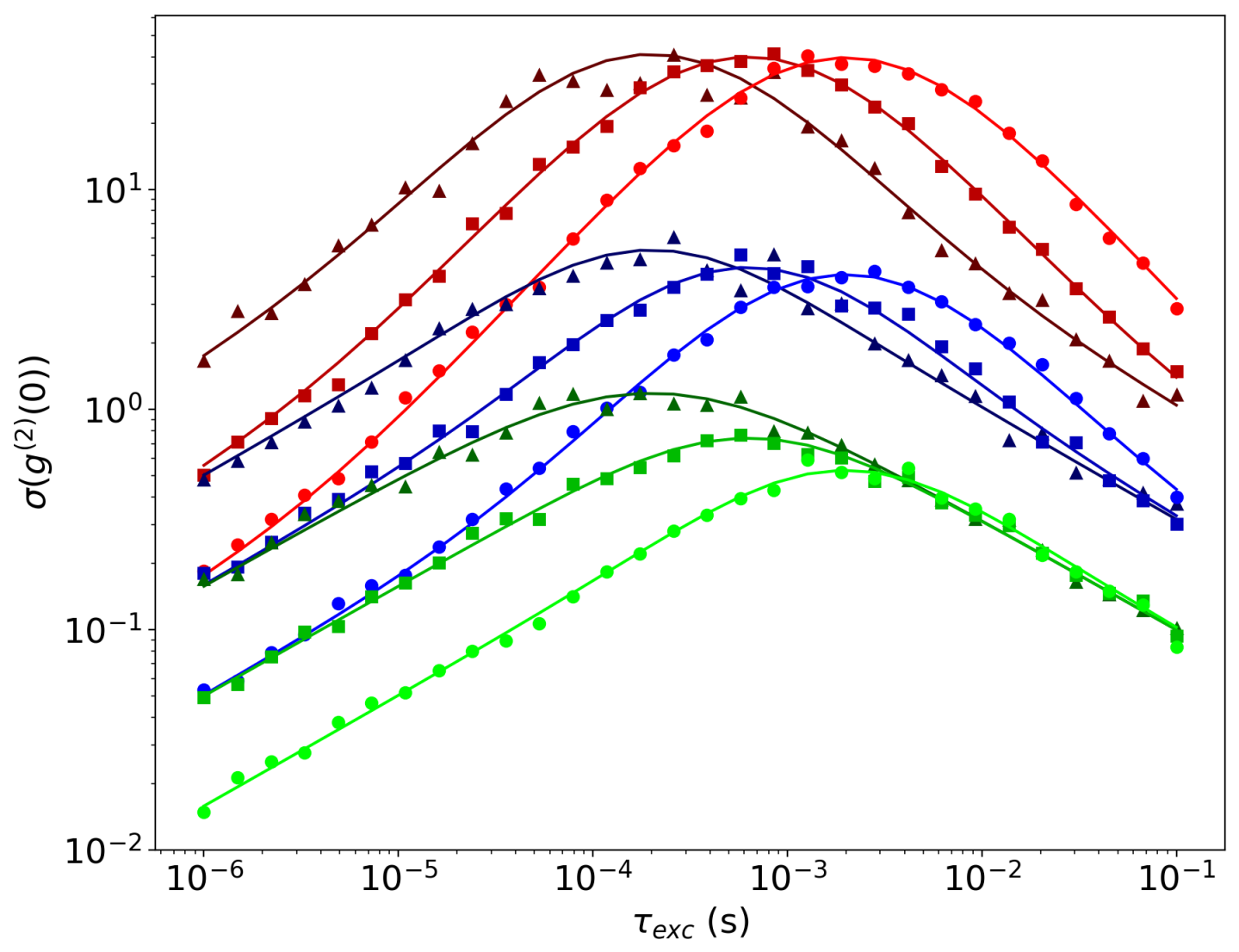

Figure 6. Compared theoretical and simulated results for $\sigma\left(g^{(2)}(0)\right)$ in the sequential DC case (Fig. 1-d) as a function of $\tau_{\text {exc }}$ for $\tau_{D}=10^{-3}$ s and $\alpha=\beta=\gamma=1$. Each color $(\mathrm{R}, \mathrm{B}, \mathrm{G})$ is associated with a value of $\tau_{2}$ in $\left(10^{-6}, 10^{-5}, 10^{-4}\right)$ s. Shapes $(\boldsymbol{\Lambda}, \boldsymbol{\square}, \bullet)$ indicate the value of $Q E$ in $(1,0.32,0.1) . \sigma$ is estimated numerically as the standard deviation of 100 experiments, for each of which $N_{\text {photons }}=1000$ are detected.

verter (mechanism b) with the following mapping:

$$
\alpha^{b} \leftrightarrow \sqrt{\alpha_{1}^{d} \alpha_{2}^{d}}, \quad \beta^{b} \leftrightarrow \gamma^{d} \frac{\alpha_{1}^{d}+\alpha_{2}^{d}-2 \sqrt{\alpha_{1}^{d} \alpha_{2}^{d}}}{\sqrt{\alpha_{1}^{d} \alpha_{2}^{d}}}, \quad \gamma^{c} \leftrightarrow \gamma^{d}, \quad \tau^{c} \leftrightarrow \tau_{2}^{d}
$$

Note that this analogy between parallel and series converters extends beyond the calculation of $g^{(2)}(0)$. For instance, it also applies to probability for the system to relax by emitting zero, one or two photons. Finally, mechanisms $\mathrm{c}$ et e can be approximated by mechanisms $\mathrm{b}$ and $\mathrm{d}$ if the intermediate transfer time is large as compared to the radiative lifetime. 
A Bayesian approach to luminescent down-conversion

\section{B. Design of experiments}

The study presented in this work offers a powerful framework for the interpretation of correlation experiments. Equation (5) provides indeed a clear insight on the influence of converters properties (lifetimes and branching ratios) and of the optical setup properties (collection and detection efficiency, dark count rate, excitation rate). We underline that the excitation rate can be related to the laser fluence $\phi$ through the absorptivity $\aleph_{A b s}$ of the sample as $\tau_{\text {exc }}^{-1}=\aleph_{A b s} \phi$, and can therefore be adjusted by changing the laser power.

Equation (5) reveals the existence of an optimal value of $\tau_{e x c}^{-}$which maximizes the correlation signal $g^{(2)}(0)-1$ (see Fig. 4). This optimal originates from a trade-off between two competing effects. On the one hand, in low-power excitation (large $\tau_{\text {exc }}$ ), the majority of photo-detections will be the result of random dark photons. Photon bunching signal is drowned out amidst the dark and the correlation function tends to 1 . On the other hand, when the excitation rate becomes large as compared to typical decay rate of the excited states, several independent emission center can be excited simultaneously leading to the superposition of unrelated signal and decreasing $g^{(2)}(0)$ towards 1 as well.

The value of this optimum occurs for an excitation level such that the number of counts on average is just the double of the noise level:

$$
\aleph_{A b s} \phi_{\mathrm{opt}} \eta_{\mathrm{LDS}} \mathrm{QE}=\frac{1}{\tau_{D}}
$$

At this illumination, the zero delay correlation function becomes:

$$
g^{(2)}(0)_{\max }-1=\eta_{\mathrm{DC}} \frac{\mathrm{QE} \tau_{D}}{4 \eta_{\mathrm{LDS}} \bar{\tau}}
$$

This expression provides for an easy determination of $g^{(2)}(0)_{\max }$ and therefore of the ratio $\eta_{\mathrm{DC}} / \eta_{\mathrm{LDS}}$, since the other parameters can be obtained independently. Indeed, $\bar{\tau}$ is estimated from the $g^{(2)}(\tau)$ measurement, $\tau_{D}$ from dark counts and QE using a calibrated source. The measurement of $\eta_{\text {LDS }}$ is generally possible, either with a photoluminescence quantum yield setup, or, if the absorptivity of the sample at excitation wavelength is known, by the relationship (8).

As a final application, we show how this study can be used for the design of experiments. Considering a converter with limited efficiency, the main difficulty is to distinguish a reduced value of the correlation function from the detection noise. According to eq.(5) and (6), it is possible to improve the signal to noise ratio by extending the duration. However, for practical purposes, the 


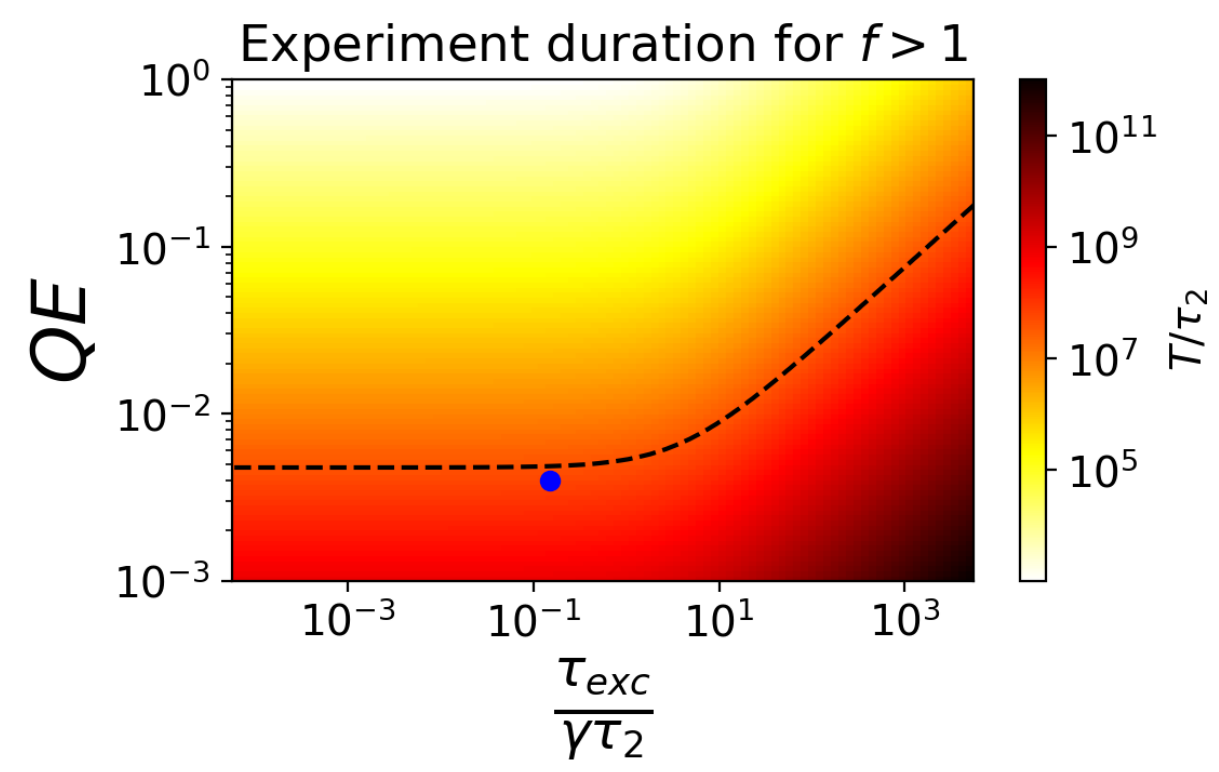

Figure 7. Minimal experiment duration to ensure $f>1$ as a function of $\tau_{\text {exc }} /\left(\gamma \tau_{2}\right)$ and $Q E$. Using the fact that $f \propto \sqrt{T}$, this heatmap can be used for any desired value of $\mathrm{f}$. In blue is an operating point used in a previous correlation study ${ }^{33}$. The dotted line acts as the lower boundary for experimental conditions to observe photon correlations with $f>1$ for a total experiment duration of $24 \mathrm{~h}$ and a promising downconversion material, taken from recent literature ${ }^{25}$.

acquisition time has to remain limited. Using our model, we can evaluate the minimal acquisition duration $T$ for the signal to be measurable

$$
f=\frac{g^{(2)}(0)-1}{\sigma\left(g^{(2)}(0)\right)}>1,
$$

The optimal value for $\mathrm{f}$, using the relationship for the optimal $g^{(2)}(0)_{\max }$, and $g^{(2)}(0)>1$, is limited by:

$$
f<\frac{Q E \eta_{\mathrm{DC}}}{2 \eta_{\mathrm{LDS}}} \frac{\sqrt{T \Delta T}}{\bar{\tau}}
$$

The results are pictured in figure 7 for a sequential converter without energy transfer (mechanism d) as a function of the apparatus properties $Q E$ and $\tau_{\text {exc }}$. For simplicity, we consider $N \tau_{D}$ to be very large as compared to $\tau_{\text {exc }}$.

The validity of this approach is tested by comparing our prediction for experiment duration with results from the literature (see Fig. 7). We first consider the $P r^{3+}$-based converters reported 
A Bayesian approach to luminescent down-conversion

in reference ${ }^{33}$, for which we find an approximate duration of $60 s$. This is coherent with the study's results, when accounting for spectral separation of photons. Indeed, for the sequential converter, being able to spectrally resolve the two transitions allows for a four-fold increase in correlation amplitude $g^{(2)}(0)-1$. In turn, this reduces experiment duration 16-fold. Considering the promising perovskite-based converters recently reported in reference ${ }^{25}$, we use our method to estimate the experimental requirements to maintain the acquisition time below $24 \mathrm{~h}$. We find for instance that the collection and detection efficiency QE has to be above $5 \times 10^{-3}$ for the signal to exceed the noise in most excitation regimes.

\section{Extensions of the method}

We have discussed the quite general character of the approach above. We discuss here how the model can be further extended to cover more situations of experimental interest. Firstly, further non-idealities affecting the conversion processes itself, such as long-range quenching effects in nanocrystals ${ }^{36}$, can be readily included in the parameters of Fig.1 as reduced lifetimes or branching ratios.

In our study, we have considered that most of our DC centers remained in the ground state, as this is the most favorable situation. Under high fluence, the occupancy of excited levels and the depopulation of the ground state depopulation can result in non-linear behaviors, such as bleaching ${ }^{25}$. The method exposed in appendix allows also, using the rate equation, can be straightforwardly adapted to take into account effects. However, we emphasize that this situation is not relevant for the devised measurement, as we have shown that optimal experimental conditions are under low illumination.

For simplicity, We have assumed that transfer steps from intermediate levels in mechanisms c and e were very fast as compared to the lifetime of the radiative state. As shown in appendix, the model can be refined to take into account a transfer function. As an example, the case where the transfer rate is proportional to the population of the initial state (exponential decay of the initial population) is treated analytically. It is found that when transfer rates are slow, zero delay correlation are lost because a time on the order of the transfer time is needed for the emission of the second photon, hence an absence of correlation at $\tau=0$. Nevertheless bunching behavior is recovered after this transfer time so that $g^{(2)}(\tau>0)>1$. A detailed study of this point beyond the scope of the present paper. 
A Bayesian approach to luminescent down-conversion

Turning to extensions of the measurement method, it is also possible to consider crosscorrelation measurements, where two detectors separated by a 50/50 beam splitter are used as a start-stop device. Such a design can be considered to circumvent the dead-time of a single detector. In the present relationships, this is done simply by changing QE into QE/2. In the case where the 2 emitted photons have distinct energies, more relationships can be obtained, as was already pointed out in ${ }^{33}$. Finally, we have only considered steady state excitation of the system. In this case, as observed here, the time scale of the first emission event does not appear explicitly in the equations. It is also possible to investigate, synchronized, pulsed excitation, and therefore use other conditional probabilities to access more system properties.

\section{CONCLUSION}

Using a Bayesian approach, we have developed analytical expressions for the correlation signal $g^{(2)}(0)$ and its standard deviation. Applying this approach to two DC systems, we have shown how the microscopic details of energy transitions could be related to the macroscopic correlation signal. Three main conclusions can be drawn from this analysis. First, despite their apparent differences, all downconverters feature analogous statistical properties. Second, correlation functions

offer an unambiguous proof for downconversion events, and can allow a quantitative estimation of the processes' efficiencies, using complementary measurements to evaluate the experimental setup properties and the absorptivity of the DC centers. Finally, the Bayesian approach establishes scaling laws which provide useful information for the design of correlation measurements, epitomizing in particular the existence of an optimal excitation rate.

\section{Appendix A: General method for a probabilistic approach to $g^{(2)}(0)$}

\section{Definition and rewriting of $g^{(2)}(0)$}

The first step in deriving a useful analytical expression for $g^{(2)}(0)$ consists in reformulating it as a ratio of photo-detection probabilities, the theory of which is rigorously described in the literature $^{35}$. We introduce

- $P(\mathrm{D}(t))$, the probability density of detecting a photon at instant $t$

- $P_{2}\left(\mathrm{D}\left(t, t^{\prime}\right)\right)$ the joint probability density of double photo-detection at instants $t$ and $t^{\prime}$ 
A Bayesian approach to luminescent down-conversion

- $P\left(\mathrm{D}\left(t^{+}\right) \mid \mathrm{D}(t)\right)$, the conditional probability density for a photo-detection to occur immediately after a first photon has been measured at instant $t$.

- $\eta$ an appropriate normalization parameter $^{35}$

And so

$$
g^{(2)}(0)=\frac{\left\langle I^{2}(t)\right\rangle}{\langle I(t)\rangle^{2}}=\frac{\eta^{2}\left\langle I^{2}(t)\right\rangle \Delta T^{2}}{(\eta\langle I(t)\rangle \Delta T)^{2}}=\frac{P_{2}\left(\mathrm{D}\left(t^{+}, t\right)\right) \Delta T^{2}}{(P(\mathrm{D}(t)) \Delta T)^{2}}=\frac{P\left(\mathrm{D}\left(t^{+}\right) \mid \mathrm{D}(t)\right)}{P(\mathrm{D}(t))}
$$

To simplify notations, from this point on $P(\mathrm{D})$ describes the average probability density of detecting a photon and $P(\mathrm{D} \mid \mathrm{D})$ the conditional probability for a photo-detection to occur immediately after a first photon has been measured. This yields

$$
g^{(2)}(0)=\frac{P(\mathrm{D} \mid \mathrm{D})}{P(\mathrm{D})}
$$

\section{Decomposing a detection event}

The problem now lies in finding an analytical expression of $P(\mathrm{D} \mid \mathrm{D})$. The general method here consists in decomposing each photo-detection event $D$ according to which type of photon was responsible for it. The decomposition is done on all mutually excluding distinguishable events. In the following, this correspond to emissions via each emitting center, but it could describe cases where a given center has several decay mechanisms (a-c cases in figure 1). To fix ideas, below, each photo-detection is caused by one and only one of :

- $A_{i}$ a first low-energy photon is emitted by center i and detected.

- $B_{i}$ a second low-energy photon is emitted by center $i$ and detected.

- $C$ a random (noise) count in the detector.

The main idea here is to decompose the detection event using mutually exclusive emission events, according to their chronological order. Notations are extended in a way that $P(\mathrm{C}) \Delta T$ is the probability of detecting a dark photon within a time interval $\Delta T$ and $P\left(\mathrm{~B}_{i}: t \mid \mathrm{A}_{j}\right) \Delta T$ is the conditional probability for detecting a photon emitted by center $\mathrm{i}$ from the second step of the conversion process within a time interval $\Delta T$ at time $t$ after a photon emitted by center $\mathrm{j}$ from the first step has been measured. Considering the probability for any first (or second) photon, it holds that:

$$
P(\mathrm{~A})=\sum_{i} P\left(\mathrm{~A}_{i}\right), \quad P(\mathrm{~B})=\sum_{i} P\left(\mathrm{~B}_{i}\right), \quad P(\mathrm{D})=P(\mathrm{~A})+P(\mathrm{~B})+P(\mathrm{C}) .
$$


The law of total probability ensures that :

$$
\begin{aligned}
P(\mathrm{D} \mid \mathrm{D})= & \sum_{i}\left(\frac{P\left(A_{i} \mid A\right) P(A)}{P(D)}+\frac{P\left(A_{i} \mid B\right) P(B)}{P(D)}\right)+\frac{P(A) P(C)}{P(D)} \\
& +\sum_{i}\left(\frac{P\left(B_{i} \mid A\right) P(A)}{P(D)}+\frac{P\left(B_{i} \mid B\right) P(B)}{P(D)}\right)+\frac{P(B) P(C)}{P(D)} \\
& +P(C)
\end{aligned}
$$

\section{Independence properties}

The final key step in deriving an analytical expression for $g^{(2)}(0)$ is simplifying conditional probabilities using properties relating to the independence of different events.

Dark photon detection are supposed to be completely random in nature, they are thus totally independent from one another and from any other detection events:

$$
\begin{gathered}
P\left(X_{i} \mid \mathrm{C}\right)=P\left(X_{i}\right) \Rightarrow \sum_{i} P\left(X_{i} \mid \mathrm{C}\right)=P(X) \\
P(\mathrm{C} \mid D)=P(\mathrm{C})=\tau_{D}^{-1}
\end{gathered}
$$

where $\mathrm{X}$ can correspond to event A or B.

Considering that all sites are independent from each other, we can express the conditional probabilities as

$$
P\left(X_{i} \mid Y\right) P(Y)=\sum_{j} P\left(X_{i} \mid Y_{j}\right) P\left(Y_{j}\right)=P\left(X_{i}\right) P(Y)+\left(P\left(X_{i} \mid Y_{i}\right)-P\left(X_{i}\right)\right) P\left(Y_{i}\right)
$$

where $\mathrm{X}$ and $\mathrm{Y}$ can correspond to event $\mathrm{A}$ or $\mathrm{B}$.

We must therefore examine conditional probabilities for transitions occurring on the same site. Just upon desexcitation, the system is unable to undergo the same transition again before going through a full cycle. The probability for the same transition to occur again immediately is thus zero. Moreover, since a detection A following a detection B originating from the same site must have undergone a random absorption in between, the two events must be independent. Finally, for a detection $B$ following a detection $A$ on the same site, the resulting probability stems from the known radiative decay of the level at stake, but actually depends on the details of the mechanism. We can therefore consider

$$
P\left(A_{i} \mid A_{i}\right)=P\left(B_{i} \mid B_{i}\right)=0 \quad P\left(A_{i} \mid B_{i}\right)=P\left(A_{i}\right)
$$


Taking these points into consideration leads to

$$
p(\mathrm{D} \mid \mathrm{D})=P(D)+\frac{\sum_{i}\left(P\left(B_{i} \mid A_{i}\right) P\left(A_{i}\right)-P\left(B_{i}\right) P\left(A_{i}\right)-P\left(B_{i}\right) P\left(B_{i}\right)-P\left(A_{i}\right) P\left(A_{i}\right)\right)}{P(D)}
$$

As will be shown in the next section, $P\left(\mathrm{~A}_{i}\right)$ and $P\left(\mathrm{~B}_{i}\right)$ both scale with $\tau_{\text {exc }}$ while $P\left(\mathrm{~B}_{i} \mid \mathrm{A}_{i}\right)$ scales with $\tau_{2}^{-1}$. In the low excitation regime $\tau_{\mathrm{exc}}>>\tau_{2}$, the first term in the parenthesis is predominant and the equation can be simplified to recover eq. 4 from the main text:

$$
p(\mathrm{D} \mid \mathrm{D})=P(D)+\frac{\sum_{i}\left(P\left(B_{i} \mid A_{i}\right) P\left(A_{i}\right)\right)}{P(D)}
$$

Finally, if all sites are assumed to be equivalent, we can write $P(\mathrm{~A})=N P\left(\mathrm{~A}_{i}\right)$ and

$$
g^{(2)}(t)=1+\frac{P_{S}(\mathrm{~B}: t \mid \mathrm{A}) P(\mathrm{~A})}{P(\mathrm{D})^{2}}
$$

where $P_{S}(\mathrm{~B}: t \mid \mathrm{A}) \Delta t=N^{-1} \sum_{i} P\left(B_{i}: t \mid A_{i}\right) \Delta t$ is the average single site conditional probability for a photon originating from the second step of the relaxation to occur within an interval $[t, t+\Delta t]$ after a first photon was detected.

\section{Appendix B: Application to the five conversion mechanisms}

In this section, we show how the previous expression can be calculated for each of the five converters considered in Fig. 1. We will consider $N$ independent and identical emission centers. The generic scheme is the following. We calculate the $\mathrm{p}(\mathrm{X})$, for $\mathrm{X}$ representing the various detection events, from detailed balance that give relationships between the different relaxation rates. Conditional probabilities can then be used to extract the term $P\left(\mathrm{~B}_{i} \mid \mathrm{A}_{i}\right)$ that is central to our formalism to calculate $g^{(2)}(t)$.

\section{Application to a series converter without energy transfer (mechanism d)}

We first consider emission centers following the processes described in Fig. 1.d. In order to evaluate the different terms of eq.(A11), we will first calculate the steady-state populations of each state, then the simple probabilities for each emission process to occur, and finally the conditional probability in the numerator.

We can estimate the steady state populations of each level with detailed balance equations, leading to

$$
\frac{\bar{N}_{g}^{d}}{\tau_{\mathrm{exc}}}=\frac{\bar{N}_{e}^{d}}{\tau_{1}^{d}}=\frac{\bar{N}_{i}^{d}}{\gamma^{d} \tau_{2}^{d}}
$$


A Bayesian approach to luminescent down-conversion

and we will neglect the depopulation of the ground state, leading to

$$
N=\bar{N}_{g}^{d}+\bar{N}_{e}^{d}+\bar{N}_{i}^{d} \simeq \bar{N}_{g}^{d}
$$

The density of probability for detecting a photon emitted by the $e \rightarrow i$ transition is thus

$$
P(\mathrm{~A})=\frac{\bar{N}_{e}^{d} \gamma^{d} \alpha_{1}^{d}}{\tau_{1}^{d}} \mathrm{QE}=\frac{N \gamma^{d} \alpha_{1}^{d} \mathrm{QE}}{\tau_{\mathrm{exc}}}
$$

and similarly

$$
P(\mathrm{~B})=\frac{\bar{N}_{i}^{d} \alpha_{2}^{d}}{\tau_{2}^{d}} \mathrm{QE}=\frac{N \gamma^{d} \alpha_{2}^{d} \mathrm{QE}}{\tau_{\text {exc }}}
$$

The density of probability for any detection to happen is given by

$$
P(\mathrm{D})=P(\mathrm{~A})+P(\mathrm{~B})+P(\mathrm{C})=\frac{N \gamma^{d} \mathrm{QE}}{\tau_{\mathrm{exc}}}\left(\alpha_{1}^{d}+\alpha_{2}^{d}\right)+\frac{1}{\tau_{D}}
$$

Finally, we estimate the average conditional probability for an event $B_{i}$ to happen a duration $t$ after a event $A_{i}$ happened. Since all sites are identical, we can estimate this probability for any site $j$ which leads to

$$
P_{S}(B: t \mid A)=P\left(B_{j}: t \mid A_{j}\right)=\frac{\alpha_{2}^{d} \mathrm{QE}}{\tau_{2}^{d}} \exp \left(-\frac{t}{\tau_{2}^{d}}\right) .
$$

Finally, the correlation function can be estimated as

$$
g^{(2)}(t)=1+\frac{\tau_{\mathrm{exc}} / N}{\tau_{2}^{d}} \frac{\gamma^{d} \alpha_{1}^{d} \alpha_{2}^{d}}{\left(\left(\gamma^{d} \alpha_{1}^{d}+\gamma^{d} \alpha_{2}^{d}\right)+\frac{\tau_{\mathrm{exc}} / N}{\tau_{D} \mathrm{QE}}\right)^{2}} \times e^{-t / \tau_{2}^{d}}
$$

\section{Application to a series converter with one-step energy transfer (mechanism e)}

We now take into account a one-step energy transfer from a first intermediate state $t$ to a second, radiative state $i$ (see Fig. 1.e). For the sake of simplicity, we describe this process with a typical transfer time $\tau_{t}^{e}$, such that the density of probability for an emitter in state $i$ to be transferred to state $i^{\prime}$ is $1 / \tau_{t}^{e}$.

Following the same steps as in the previous section, we estimate readily

$$
\frac{N}{\tau_{\mathrm{exc}}} \simeq \frac{\bar{N}_{e}^{e}}{\tau_{1}^{e}}=\frac{\bar{N}_{t}^{e}}{\gamma^{e} \tau_{t}^{e}}=\frac{\bar{N}_{i}^{e}}{\gamma^{e} \tau_{2}^{e}}
$$

and

$$
P(\mathrm{~A})=N \frac{\gamma^{e} \alpha_{1}^{e} \mathrm{QE}}{\tau_{\mathrm{exc}}}, \quad P(\mathrm{~B})=N \frac{\gamma^{e} \alpha_{2}^{e} \mathrm{QE}}{\tau_{\mathrm{exc}}}, \quad P(\mathrm{C})=\frac{1}{\tau_{D}}
$$


We now turn to the calculation of conditional probability. Following the same steps as before, we need to estimate the probability for a center to emit a second photon at time $t$ knowing that a first photon has been emitted a time 0 . This process requires that the system is transferred from the $t$ state to the $i$ state, kept in this state until time $t$, and only then relaxes by emitting a photon which is collected and detected. We will consider that the density of probability for a particle in state $t$ to be transferred is $\left(\tau_{t}^{e}\right)^{-1}$, but a more complex transfer function can easily be implemented. This chain of events can be expressed as :

$$
\begin{aligned}
P_{S}(B: t \mid A) & =\frac{\alpha_{2}^{e} \mathrm{QE}}{\tau_{2}^{e}} \int_{0}^{t} d u \frac{\exp \left(-\frac{u}{\tau_{t}^{e}}\right)}{\tau_{t}^{e}} \times \exp \left(-\frac{t-u}{\tau_{2}^{e}}\right) \\
& =\frac{\alpha_{2}^{e} \mathrm{QE}}{\tau_{2}^{e}-\tau_{t}^{e}}\left(e^{-t / \tau_{2}^{e}}-e^{-t / \tau_{t}^{e}}\right)
\end{aligned}
$$

Note that this expression is actually a smooth function (that can be written as a Hyperbolic Sine Cardinal) and can be defined by continuity when $\tau_{t}^{e}=\tau_{2}^{e}$.

We can therefore estimate the autocorrelation function as

$$
g^{(2)}(t)=1+\frac{\tau_{\mathrm{exc}} / N}{\tau_{2}^{e}-\tau_{t}^{e}} \frac{\gamma^{e} \alpha_{1}^{e} \alpha_{2}^{e}}{\left(\gamma^{e} \alpha_{1}^{e}+\gamma^{e} \alpha_{2}^{e}+\frac{\tau_{\mathrm{exc}} / N}{\tau_{D} \mathrm{QE}}\right)^{2}}\left(e^{-t / \tau_{2}^{e}}-e^{-t / \tau_{t}^{e}}\right)
$$

In the general case, this expression tends towards 1 as $t \rightarrow 0^{+}$. However, the autocorrelation function increases above 1 with time, reaches a maximum value proportional to $\gamma^{e} \alpha_{1}^{e} \alpha_{2}^{e}$ before decreasing back towards 1 . Therefore, in this case as well, an correlation value exceeding 1 is indeed a direct signature of down conversion. Furthermore, if the transfer time is much shorter than the radiative lifetime (which is the case in real systems ${ }^{30}$ ), we recover an expression similar to the previous one:

$$
g^{(2)}(t) \underset{\tau_{t}^{e} \ll \tau_{2}^{e}}{\rightarrow} 1+\frac{\tau_{\mathrm{exc}} / N}{\tau_{2}^{e}} \frac{\gamma^{e} \alpha_{1}^{e} \alpha_{2}^{e}}{\left(\gamma^{e} \alpha_{1}^{e}+\gamma^{e} \alpha_{2}^{e}+\frac{\tau_{\mathrm{exc}} / N}{\tau_{D} \mathrm{QE}}\right)^{2}} e^{-t / \tau_{2}^{e}}
$$

\section{Application to a parallel converter with one-step energy transfer (mechanisms a and b)}

In parallel converters, two radiative states (labeled $i_{1}$ and $i_{2}$ ) can be populated simultaneously on the same site, and the emission resulting in event $A$ can originate indistinctly from any of these states. The expression of steady-state populations is similar to the previous calculations:

$$
\frac{N}{\tau_{\mathrm{exc}}} \simeq \frac{N_{e}^{a}}{\tau_{t}^{a}}=\frac{N_{i 1}^{a}}{\left(\gamma^{a}+\beta^{a}\right) \tau_{1}^{a}}=\frac{N_{i 2}^{a}}{\gamma^{a} \tau_{2}^{a}}
$$


A Bayesian approach to luminescent down-conversion

and

$$
P(\mathrm{D})=\frac{N}{\tau_{\mathrm{exc}}}\left(\left(\gamma^{a}+\beta^{a}\right) \alpha_{1}^{a}+\gamma^{a} \alpha_{2}^{a}\right) \mathrm{QE}+\frac{1}{\tau_{D}}
$$

However, as compared to the case of series converters, two mutually exclusive situations have to be taken into account. The first relaxation originates indeed either from the $i_{1} \rightarrow g$ transition and the second transition can only result from the $i_{2} \rightarrow g$ transition, or vice versa. We distinguish these cases in the expression of the correlation function by expliciting which transition is at stake:

$$
g^{(2)}(t)=1+\frac{P_{S}(B 2: t \mid A 1) P(A 1)+P_{S}(B 1: t \mid A 2) P(A 2)}{P(\mathrm{D})^{2}}
$$

We will estimate each of these terms. Event $A 1$ corresponds to the emission and detection of a photon from transition 1 in any system, before a photon from transition 2 is emitted in this system. This requires that both states are populated in the first place, and that state 2 remains populated while state 1 relaxes radiatively. The corresponding probability can be expressed as

$$
P(A 1)=\frac{\alpha_{1}^{a} \mathrm{QE}}{\tau_{1}^{a}} \frac{\gamma^{a}}{\gamma^{a}+\beta^{a}} N_{i 1} \int_{0}^{\infty} d t e^{-t / \tau_{2}^{a}} \frac{e^{-t / \tau_{1}^{a}}}{\tau_{1}^{a}}=\frac{N}{\tau_{\mathrm{exc}}} \frac{\tau_{2}^{a}}{\tau_{1}^{a}+\tau_{2}^{a}} \gamma^{a} \alpha_{1}^{a} \mathrm{QE}
$$

Similarly

$$
P(A 2)=\frac{\alpha_{2}^{a} \mathrm{QE}}{\tau_{2}^{a}} N_{i 2} \int_{0}^{\infty} d t e^{-t / \tau_{1}^{a}} \frac{e^{-t / \tau_{2}^{a}}}{\tau_{2}^{a}}=\frac{N}{\tau_{\text {exc }}} \frac{\tau_{1}^{a}}{\tau_{1}^{a}+\tau_{2}^{a}} \gamma^{a} \alpha_{2}^{a} \mathrm{QE}
$$

In the same way, event $B 1$ corresponds to the transition 1 taking place while transition 2 has already happened. The corresponding probability can be expressed as

$$
\begin{aligned}
& P(B 1)=\frac{\alpha_{1}^{a} \mathrm{QE}}{\tau_{1}^{a}} \frac{\gamma^{a}}{\gamma^{a}+\beta^{a}} N_{i 1} \int_{0}^{\infty} d t\left(1-e^{-t / \tau_{2}^{a}}\right) \frac{e^{-t / \tau_{1}^{a}}}{\tau_{1}^{a}}=\frac{N}{\tau_{\mathrm{exc}}} \frac{\tau_{1}^{a}}{\tau_{1}^{a}+\tau_{2}^{a}} \gamma^{a} \alpha_{1}^{a} \mathrm{QE} \\
& P(B 2)=\frac{\alpha_{2}^{a} \mathrm{QE}}{\tau_{2}^{a}} N_{i 2} \int_{0}^{\infty} d t\left(1-e^{-t / \tau_{1}^{a}}\right) \frac{e^{-t / \tau_{2}^{a}}}{\tau_{2}^{a}}=\frac{N}{\tau_{\mathrm{exc}}} \frac{\tau_{2}^{a}}{\tau_{1}^{a}+\tau_{2}^{a}} \gamma^{a} \alpha_{2}^{a} \mathrm{QE}
\end{aligned}
$$

To evaluate the conditional probabilities, we proceed as before, leading to:

$$
P_{S}(B 2: t \mid A 1)=\frac{\alpha_{2}^{a} \mathrm{QE}}{\tau_{2}^{a}} \exp \left(-\frac{t}{\tau_{2}^{a}}\right) \quad P_{S}(B 1: t \mid A 2)=\frac{\alpha_{1}^{a} \mathrm{QE}}{\tau_{1}^{a}} \exp \left(-\frac{t}{\tau_{1}^{a}}\right) .
$$

This results in the final expression for mechanism a:

$$
g^{(2)}(t)=1+\frac{\tau_{\mathrm{exc}} / N}{\tau_{1}^{a}+\tau_{2}^{a}} \frac{\gamma \alpha_{1}^{a} \alpha_{2}^{a}}{\left(\left(\gamma^{a}+\beta^{a}\right) \alpha_{1}^{a}+\gamma^{a} \alpha_{2}^{a}+\frac{\tau_{\mathrm{exc}} / N}{\tau_{D} \mathrm{QE}}\right)^{2}}\left(e^{-t / \tau_{2}^{a}}+e^{-t / \tau_{1}^{a}}\right)
$$

For mechanism $b, \alpha_{1}=\alpha_{2}=\alpha^{b}$ and $\tau_{1}=\tau_{2}=\tau^{b}$, leading to

$$
g^{(2)}(t)=1+\frac{\tau_{\mathrm{exc}} / N}{\tau^{b}} \frac{\gamma^{b}\left(\alpha^{b}\right)^{2}}{\left(\left(2 \gamma^{b}+\beta^{b}\right) \alpha^{b}+\frac{\tau_{\mathrm{exc}} / N}{\tau_{D} \mathrm{QE}}\right)^{2}} \times e^{-t / \tau^{b}}
$$




\section{Application to a parallel converter with two-steps energy transfer (mechanisms c)}

Finally, we turn to the case of a parallel converter with a two step energy transfer. The steadystate populations can be estimated as before from detailed balance considerations:

$$
\frac{N}{\tau_{\mathrm{exc}}} \simeq \frac{N_{e}^{c}}{\tau_{t}^{c}}=\frac{N_{t}^{c}}{\left(\gamma^{c}+\beta^{c}\right) \tau_{t}^{c}}=\frac{N_{i 1}^{c}}{\left(\gamma^{c}+\beta^{c}\right) \tau^{c}}=\frac{N_{i 2}^{c}}{\gamma^{c} \tau^{c}}
$$

leading to

$$
P(\mathrm{D})=\frac{N}{\tau_{\mathrm{exc}}}\left(\left(2 \gamma^{c}+\beta^{c}\right) \alpha^{c}\right) \mathrm{QE}+\frac{1}{\tau_{D}}
$$

We consider the same decomposition as in the previous section

$$
g^{(2)}(t)=1+\frac{P_{S}(B 2: t \mid A 1: 0) P(A 1)+P_{S}(B 1: t \mid A 2: 0) P(A 2)}{P(\mathrm{D})^{2}}
$$

and we evaluate the probability including the transfer processes as in mechanism e :

$$
\begin{aligned}
P(A 1) & =\frac{\alpha_{1} \mathrm{QE}}{\tau^{c}} \frac{\gamma^{c}}{\gamma^{c}+\beta^{c}} N_{i 1} \int_{0}^{\infty} d t e^{-t / \tau^{c}} \frac{1}{\tau^{c}} \int_{0}^{t} d u \frac{e^{-u / \tau_{t}}}{\tau_{t}} e^{-(t-u) / \tau^{c}} \\
& =\frac{N \alpha_{1} \gamma^{c} \mathrm{QE}}{\tau_{\mathrm{exc}}} \frac{\tau^{c}}{2\left(\tau_{t}+\tau^{c}\right)} \\
P(A 2) & =\frac{N \alpha_{2} \gamma^{c} \mathrm{QE}}{\tau_{\mathrm{exc}}}\left(1-\frac{\tau^{c}}{2\left(\tau_{t}+\tau^{c}\right)}\right) \\
P(B 1) & =\frac{N \alpha_{1} \gamma^{c} \mathrm{QE}}{\tau_{\mathrm{exc}}}\left(1-\frac{\tau^{c}}{2\left(\tau_{t}+\tau^{c}\right)}\right) \\
P(B 2) & =\frac{N \alpha_{2} \gamma^{c} \mathrm{QE}}{\tau_{\mathrm{exc}}} \frac{\tau^{c}}{2\left(\tau_{t}+\tau^{c}\right)}
\end{aligned}
$$

Finally, conditional probabilities are expressed as before:

$$
P_{S}(B 2: t \mid A 1)=\frac{\alpha_{2}^{a} \mathrm{QE}}{\tau^{c}} \exp \left(-\frac{t}{\tau^{c}}\right) \quad P_{S}(B 1: t \mid A 2)=\alpha_{1}^{a} \mathrm{QE} \frac{e^{-t / \tau^{c}}-e^{-t / \tau_{t}}}{\tau^{c}-\tau_{t}}
$$

leading to the expression of the correlation function

$$
g^{(2)}(0)=1+\frac{\tau_{\mathrm{exc}} / N}{2\left(\tau_{t}+\tau^{c}\right)} \frac{\gamma^{c}\left(\alpha^{c}\right)^{2}}{\left(\left(\left(2 \gamma^{c}+\beta^{\mathrm{c}}\right) \alpha^{\mathrm{c}}\right)+\frac{\tau_{\mathrm{exc}} / \mathrm{N}}{\mathrm{QE} \tau_{\mathrm{D}}}\right)^{2}}\left(e^{-t / \tau^{c}}+\frac{\tau^{c}+2 \tau_{t}}{\tau^{c}-\tau_{t}}\left(e^{-t / \tau^{c}}-e^{-t / \tau_{t}}\right)\right)
$$

For very fast transfer times as compared to the radiative lifetime, as discussed for mechanism e, we recover the same expression as mechanism b

$$
g^{(2)}(0)=1+\frac{\tau_{\mathrm{exc}} / N}{\tau^{c}} \frac{\gamma^{c}\left(\alpha^{c}\right)^{2}}{\left(\left(\left(2 \gamma^{c}+\beta^{c}\right) \alpha^{c}\right)+\frac{\tau_{\mathrm{exc}} / \mathrm{N}}{\mathrm{QE} \tau_{\mathrm{D}}}\right)^{2}} \times e^{-t / \tau^{c}}
$$


A Bayesian approach to luminescent down-conversion

\section{REFERENCES}

${ }^{1}$ W. van Sark, A. Meijerink, R. Schropp, J. van Roosmalen, and E. Lysen, "Enhancing solar cell efficiency by using spectral converters," Solar Energy Materials and Solar Cells 87, 395-409 (2005).

${ }^{2}$ B. Richards and K. McIntosh, "Overcoming the poor short wavelength spectral response of $\mathrm{CdS} / \mathrm{CdTe}$ photovoltaic modules via luminescence down-shifting: ray-tracing simulations," Progress in Photovoltaics: Research and Applications 15, 27-34 (2007).

${ }^{3}$ X. Huang, S. Han, W. Huang, and X. Liu, "Enhancing solar cell efficiency: the search for luminescent materials as spectral converters," Chem. Soc. Rev. 42, 173-201 (2013).

${ }^{4}$ J. Day, S. Senthilarasu, and T. K. Mallick, "Improving spectral modification for applications in solar cells: A review," Renewable Energy 132, 186-205 (2019).

${ }^{5}$ C. Strümpel, M. McCann, G. Beaucarne, V. Arkhipov, A. Slaoui, V. Švrček, C. del Cañizo, and I. Tobias, "Modifying the solar spectrum to enhance silicon solar cell efficiency-An overview of available materials," Solar Energy Materials and Solar Cells 91, 238-249 (2007).

${ }^{6}$ H. Shpaisman, O. Niitsoo, I. Lubomirsky, and D. Cahen, "Can up- and down-conversion and multi-exciton generation improve photovoltaics?" Solar Energy Materials and Solar Cells 92, 1541-1546 (2008).

${ }^{7}$ E. Klampaftis, D. Ross, K. R. McIntosh, and B. S. Richards, "Enhancing the performance of solar cells via luminescent down-shifting of the incident spectrum: A review," Solar Energy Materials and Solar Cells 93, 1182-1194 (2009).

${ }^{8}$ H. J. Hovel, R. T. Hodgson, and J. M. Woodall, “The effect of fluorescent wavelength shifting on solar cell spectral response," Solar Energy Materials 2, 19-29 (1979).

${ }^{9}$ T. Fix, A. Nonat, D. Imbert, S. D. Pietro, M. Mazzanti, A. Slaoui, and L. J. Charbonnière, "Enhancement of silicon solar cells by downshifting with Eu and Tb coordination complexes," Progress in Photovoltaics: Research and Applications 24, 1251-1260 (2016), _eprint: https://onlinelibrary.wiley.com/doi/pdf/10.1002/pip.2785.

${ }^{10}$ W. Shockley and H. J. Queisser, "Detailed Balance Limit of Efficiency of p-n Junction Solar Cells," Journal of Applied Physics 32, 510-519 (1961), publisher: American Institute of Physics.

${ }^{11}$ T. Trupke, M. A. Green, and P. Würfel, "Improving solar cell efficiencies by down-conversion of high-energy photons," Journal of Applied Physics 92, 1668 (2002).

${ }^{12}$ T. Trupke, M. A. Green, and P. Würfel, "Improving solar cell efficiencies by up-conversion of 
sub-band-gap light,” Journal of Applied Physics 92, 4117-4122 (2002).

${ }^{13}$ R. Schaller and V. Klimov, "High Efficiency Carrier Multiplication in PbSe Nanocrystals: Implications for Solar Energy Conversion,” Physical review letters 92, 186601 (2004).

${ }^{14}$ V. Švrček, A. Slaoui, and J. C. Muller, "Silicon nanocrystals as light converter for solar cells," Thin Solid Films Proceedings of Symposium D on Thin Film and Nano-Structured Materials for Photovoltaics, of the E-MRS 2003 Spring Conference, 451-452, 384-388 (2004).

${ }^{15}$ G. Chen, C. Yang, and P. N. Prasad, "Nanophotonics and Nanochemistry: Controlling the Excitation Dynamics for Frequency Up- and Down-Conversion in Lanthanide-Doped Nanoparticles," Accounts of Chemical Research 46, 1474-1486 (2013), publisher: American Chemical Society.

${ }^{16}$ M. L. Böhm, T. C. Jellicoe, M. Tabachnyk, N. J. L. K. Davis, F. Wisnivesky-Rocca-Rivarola, C. Ducati, B. Ehrler, A. A. Bakulin, and N. C. Greenham, "Lead Telluride Quantum Dot Solar Cells Displaying External Quantum Efficiencies Exceeding 120\%,” Nano Letters 15, 7987-7993 (2015), publisher: American Chemical Society.

${ }^{17}$ I. Paci, J. C. Johnson, X. Chen, G. Rana, D. Popovic, D. E. David, A. J. Nozik, M. A. Ratner, and J. Michl, "Singlet Fission for Dye-Sensitized Solar Cells: Can a Suitable Sensitizer Be Found?" Journal of the American Chemical Society 128, 16546-16553 (2006), publisher: American Chemical Society.

${ }^{18}$ L. H. Slooff, R. Kinderman, A. R. Burgers, N. J. Bakker, J. a. M. van Roosmalen, A. Büchtemann, R. Danz, and M. Schleusener, "Efficiency Enhancement of Solar Cells by Application of a Polymer Coating Containing a Luminescent Dye," Journal of Solar Energy Engineering 129, 272-276 (2007), publisher: American Society of Mechanical Engineers Digital Collection.

${ }^{19}$ J. Lee, P. Jadhav, P. D. Reusswig, S. R. Yost, N. J. Thompson, D. N. Congreve, E. Hontz, T. Van Voorhis, and M. A. Baldo, "Singlet Exciton Fission Photovoltaics," Accounts of Chemical Research 46, 1300-1311 (2013), publisher: American Chemical Society.

${ }^{20} \mathrm{Z}$. Wang and A. Meijerink, "Dye-Sensitized Downconversion," The Journal of Physical Chemistry Letters 9, 1522-1526 (2018), publisher: American Chemical Society.

${ }^{21}$ R. T. Wegh, H. Donker, E. V. D. van Loef, K. D. Oskam, and A. Meijerink, "Quantum cutting through downconversion in rare-earth compounds," Journal of Luminescence 87-89, 1017-1019 (2000).

${ }^{22}$ B. M. v. d. Ende, L. Aarts, and A. Meijerink, "Near-Infrared Quantum Cutting for Photovoltaics," Advanced Materials 21, 3073-3077 (2009), _eprint: https://onlinelibrary.wiley.com/doi/pdf/10.1002/adma.200802220. 
A Bayesian approach to luminescent down-conversion

${ }^{23}$ X. Y. Huang and Q. Y. Zhang, "Near-infrared quantum cutting via cooperative energy transfer in Gd2O3:Bi3+,Yb3+ phosphors," Journal of Applied Physics 107, 063505 (2010), publisher: American Institute of Physics.

${ }^{24}$ L. d. A. Florêncio, L. A. Gómez-Malagón, B. C. Lima, A. S. L. Gomes, J. A. M. Garcia, and L. R. P. Kassab, "Efficiency enhancement in solar cells using photon down-conversion in $\mathrm{Tb} / \mathrm{Yb}$ doped tellurite glass," Solar Energy Materials and Solar Cells 157, 468-475 (2016).

${ }^{25}$ C. S. Erickson, M. J. Crane, T. J. Milstein, and D. R. Gamelin, "Photoluminescence Saturation in Quantum-Cutting $\mathrm{Yb}^{3+}$-Doped $\mathrm{CsPb}\left(\mathrm{Cl}_{1-x} \mathrm{Br}_{x}\right)_{3}$ Perovskite Nanocrystals: Implications for Solar Downconversion,” The Journal of Physical Chemistry C 123, 12474-12484 (2019).

${ }^{26}$ B. Fan, C. Chlique, O. Merdrignac-Conanec, X. Zhang, and X. Fan, "Near-Infrared Quantum Cutting Material Er3+/Yb3+ Doped La2O2S with an External Quantum Yield Higher than 100\%," The Journal of Physical Chemistry C 116, 11652-11657 (2012), publisher: American Chemical Society.

${ }^{27}$ D. C. Yu, F. T. Rabouw, W. Q. Boon, T. Kieboom, S. Ye, Q. Y. Zhang, and A. Meijerink, "Insights into the energy transfer mechanism in Ce3+ Yb3+ codoped YAG phosphors," Physical Review B 90, 165126 (2014).

${ }^{28}$ K. Wu, G. Liang, Q. Shang, Y. Ren, D. Kong, and T. Lian, "Ultrafast Interfacial Electron and Hole Transfer from $\mathrm{CsPbr}_{3}$ Perovskite Quantum Dots," Journal of the American Chemical Society 137, 12792-12795 (2015).

${ }^{29}$ T. J. Milstein, D. M. Kroupa, and D. R. Gamelin, "Picosecond Quantum Cutting Generates Photoluminescence Quantum Yields Over 100\% in Ytterbium-Doped $\mathrm{CsPbCl}_{3}$ Nanocrystals," Nano Letters 18, 3792-3799 (2018).

${ }^{30}$ J.-M. Meijer, L. Aarts, B. M. van der Ende, T. J. H. Vlugt, and A. Meijerink, "Downconversion for solar cells in YF 3 : Nd 3 +, Yb 3 +," Physical Review B 81 (2010), 10.1103/PhysRevB.81.035107.

${ }^{31}$ N. Geacintov, M. Pope, and F. Vogel, "Effect of Magnetic Field on the Fluorescence of Tetracene Crystals: Exciton Fission,” Physical Review Letters 22, 593-596 (1969).

${ }^{32}$ M. Einzinger, T. Wu, J. F. Kompalla, H. L. Smith, C. F. Perkinson, L. Nienhaus, S. Wieghold, D. N. Congreve, A. Kahn, M. G. Bawendi, and M. A. Baldo, "Sensitization of silicon by singlet exciton fission in tetracene," Nature 571, 90-94 (2019).

${ }^{33}$ M. de Jong, A. Meijerink, and F. T. Rabouw, "Non-Poissonian photon statistics from macroscopic photon cutting materials," Nature Communications 8, 15537 (2017). 
A Bayesian approach to luminescent down-conversion

${ }^{34}$ M. Fox, Quantum Optics: An Introduction (OUP Oxford, 2006).

${ }^{35}$ L. Mandel and E. Wolf, Optical Coherence and Quantum Optics (Cambridge University Press, Cambridge, 1995).

${ }^{36}$ S. Fischer, N. D. Bronstein, J. K. Swabeck, E. M. Chan, and A. P. Alivisatos, "Precise Tuning of Surface Quenching for Luminescence Enhancement in Core-Shell Lanthanide-Doped Nanocrystals," Nano Letters 16, 7241-7247 (2016).

Data available on request from the authors 